\title{
ShigelDysent efficacy in alleviating clinical sign and symptoms
} prevailing in Shigellosis

Tasneem Qureshi ${ }^{1}$, Aftab Saeed ${ }^{1}$, Khan Usmanghani ${ }^{1}$, Hafiz Muhammad Asif ${ }^{2}$ and Syed Faisal Zaidi ${ }^{3,4^{*}}$

${ }^{1}$ Department of Basic Clinical Sciences, Faculty of Eastern Medicine, Hamdard University Karachi, Pakistan

${ }^{2}$ Department of Eastern Medicine \& Surgery, Faculty of Medical \& Health Sciences, The University of Poonch, Rawalakot, AJ\&K, Pakistan ${ }^{3}$ Department of Basic Medical Sciences, College of Medicine, King Saud bin Abdulaziz University of Health Sciences, Jeddah 21423, Kingdom of Saudi Arabia

${ }^{4}$ Department of Gastroenterology, Graduate School of Medical \& Pharmaceutical Sciences, University of Toyama, Toyama 930-0194, Japan

\section{Abstract}

Background: Bacillary dysentery (Shigellosis) is an intestinal infection caused by the Shigella spp is potentially contagious for human health. The significant hallmark of Shigellosis is the bloody diarrhea particularly in developing countries with poor quality hygiene. Shigella species are accountable for a morbidity as well as mortality and poses threat to kids younger than five years and elder people with an undermined immune system. However, Shigella quickly spread in place having suffocated crowded dwellings and is transmitted directly by fecal material or through contaminated food or water.

Methods: A comparative study was undertaken to determine the efficacy of ShigelDysent (Test drug) in comparison with Ciprofloxacin (Control drug) in alleviating the clinical sign and symptoms of Shigellosis. The research was undertaken among age group 25-45 years in 2010-2013. Shigellosis was recorded in 250 patients dividing them into two parallel arm groups; 125 for test and 125 for control group each without consideration of the socio-economic status.

Results: Results showed that the medicinal plant coded formulation ShigelDysent exhibit significant ( $\mathrm{p}<$ 0.029) efficacy in relieving the clinical features for sign and symptoms of Shigellosis comparable with that of standard drug Ciprofloxacin.

Conclusion: ShigelDysent is effective in the in the treatment and management of Shigellosis. However further studies on large scale are suggested to find out the pharmacological action of ShigelDysent.

\section{Background}

Shigella is entero-invasive bacteria which causes classical bacillary dysentery. The principal species causing gastroenteritis are Shigella dysenteriae, Shigella flexneri and Shigella sonnei which are found with varying epidemiological prevalence in different countries. All these species lead to a similar syndrome as a result of damage to the intestinal mucosa. Some strains of Shigella dysenteriae also secrete a cytotoxin affecting vascular endothelium. The organism is spread from person to person and only small numbers are afflicted and need to be taken to cure the illness for example $<200$, compared to 104 for Campylobacter and $>105$ for Salmonella). Bacillary dysentery is far more prevalent in the developing world because of poor hygienic conditions [13]. The term dysentery is specifically used for the passage of stool with pain and cramps. Dysentery is of two kinds, one is limited to rectum while the other is confined to transverse colon, which is in contact or proximal to the rectum. Difference in both is obvious as dysentery occurs due to abrasion and muco-purulent discharge. There is no discomfort found in the rectum, tenesmus is less, excretion is possible by least tenesmus and patient remains asymptomatic. In rectal dysentery, defecation is painful with small fecal matter and tenesmus and patient suffer with fatigue easily [4]. It is colonic dysentery; sometimes inner portion of intestinal epithelial lining gets affected. In this case intestinal membrane becomes edematous that results in tissue death. These membranous scales are excreted out in stool [5]. Bacillary dysentery results from Shigella species, provokes passage of fecal blood and mucus. On reaching the large intestine the organism passes through the cell lining via $\mathrm{M}$ cells that superimpose the lymphoid nodules [6-8].

In view of the fact that due to bacterial resistance the current global trends and direction started to treat diarrheal diseases with herbal drugs. As such it is a need to strategically utilize efficacy based herbal medicine to eliminate diarrhea. In order to prove the effectiveness of medicinal plants on scientific and clinical basis, we have designed and report here with the effectiveness of herbal coded formulation "ShigelDysent" for the eradication of Shigella species and it has shown significant results in a cited recent report [9]. In the present study, we have focused on the effectiveness of "ShigelDysent" in alleviating the clinical manifestations of Shigellosis.

\section{Materials and Methods}

The research study is an experimental, randomized, clinical trial conducted during 2010-2013. The trials were executed in Shifa-ulMulk Memorial Hospital for Eastern Medicine (SUMMH), Hamdard University, Pehlwan Goth, Safoora Goth and Patel Para, Karachi, Pakistan. All patients were randomly assigned either Ciprofloxacin or ShigelDysent and divided into two groups according to treatment regimen i. e., test group and control group respectively. Clinical evaluation of the disease and also rectal swab cultures were made before and after treatment for seven days. Before starting the specific therapy, rectal swabs were obtained and transferred and immediately inoculated on to MacConkey and S.S. agar plates. Antimicrobial

"Corresponding Author: Dr. Syed Faisal Zaidi, College of Medicine, King Saud bin Abdulaziz University of Health Sciences, Jeddah 21423, Kingdom of Saudi Arabia, Tel: +966-222-45766; E-mail: sfaisalhz@gmail.com; zaidis@ksau.edu.sa

Citation: Qureshi T, Saeed A, Usmanghani K, Asif HM, Zaidi SF (2015) ShigelDysent efficacy in alleviating clinical sign and symptoms prevailing in Shigellosis. Int J Gastroenterol Disord Ther 2: 114. doi: http://dx.doi. org/10.15344/2393-8498/2015/114

Copyright: (c) 2015 Qureshi et al. This is an open-access article distributed under the terms of the Creative Commons Attribution License, which permits unrestricted use, distribution, and reproduction in any medium, provided the original author and source are credited. 
Citation: Qureshi T, Saeed A, Usmanghani K, Asif HM, Zaidi SF (2015) ShigelDysent efficacy in alleviating clinical sign and symptoms prevailing in Shigellosis. Int J Gastroenterol Disord Ther 2: 114. doi: http://dx.doi.org/10.15344/2393-8498/2015/114

therapy was started without awaiting the results of stool cultures. Patients were withdrawn from the study if their stool cultures did not confirm the diagnosis of the presence of Shigella organisms. Research proforma was filled for documentation by the principal investigator that included the complete clinical data including the sign and symptoms of the bacillary dysentery (Shigellosis) and patient follow up to evaluate the drug efficacy.

\section{Sample size}

Sample size comprises of 250 cases each of test group (125) and control group (125) prescribed and administered "ShigelDysent" as test and "Ciprofloxacin" as control drug.

\section{Selection of herbal drugs for Shigellosis}

The medicinal plants effective for shigellosis were selected after thorough review of literature search on Herbal Pharmacopeias and research articles available in electronic journals. The medicinal plants showing remarkable ethno botanical and scientific evidences for the treatment of dysentery were selected namely Phyllanthus emblica L., Aegle marmelos L., Holerrhena antidysenterica L., Myrtus communis L., Polygonum bistorta L., Citrus aurantifolia L. in powder form to be used as capsules. All these phyto-pharmaceutical ingredients were grinded and aqueous alcoholic extract were filled in the capsule.

\section{Identification and preparation}

The selected drugs for bacillary dysentery (Shigellosis) were purchased from the local market at Jodia Bazar. All the six herbal drugs were powdered separately into fine quality and filter through 100 number sieves in pharmacy at Hamdard Al-Majeed College of Eastern Medicine (HACEM), Hamdard University.

All the herbal drugs are separately weighed as per dosage in $500 \mathrm{mg}$ capsule: Myrtus communis L. (Fruit of Hub-ul-Aas powder)-150mg, Aegle marmelos L. (Fruit of Belgiri powder)-100mg, Polygonum bistorta L. (Bikh Anjibar powder)-75mg, Phyllanthus emblica L. (Fruit of Aamla powder)-75mg, Holarrhena antidysenterica L. (Maroor phalli powder)-50mg, Citrus aurantifolia L. (Post Turang powder)50mg. All the herbal drugs were cleaned and examined for their impurities and adulterations in pharmacy of HAMCEM. The herbal drugs were weighed according to the dosage in formulation after powdered in grinding machine and filtered through 100 number sieves. The identity of the herbal drugs samples were identified and authenticated by Prof. Dr. Iqbal Azhar, Chairman, Department of Pharmacognosy, University of Karachi on 1st January 2010.

\section{Statistical analysis}

The SPSS for windows version 17 was applied for the statistical analysis of data for verification and to check for range and consistency with customized data entry and XP software. After clinical follow-up the data so generated was included electronically and recorded. All the data was twice recorded in programs such as SPSS, XP Microsoft. Since the observation period was 7-10 days, current status of the disease along with new out comings of the disease episodes was also recorded. Non-parametric analysis was done by the use of the Wilcoxon rank sum test and Mann-Whitney test for assessment between the groups, to compare two paired groups proportion T-test, to compare three or more unmatched groups one way ANOVA was applied to assess the statistical significance. Clinical success for patients treated with ShigelDysent was predicted at $95 \%$.

\section{Results}

A total of 250 patients were assigned test and control therapy by dividing them into two groups i.e., test and control group. Patients in the test group were prescribed ShigelDysent $(500 \mathrm{mg})$ while patients in control group were given Ciprofloxacin $(500 \mathrm{mg}$ ) for 7 days.

The major clinical manifestation of Shigellosis is the increase of stool frequency and disturbance in stool consistency. Patients in the test group showed marked improvement both in stool frequency and consistency which is comparable with that of standard drug Ciprofloxacin [Table 1 and 2], exhibiting that ShigelDysent is significant $(p<0.005)$ in the treatment of clinical manifestation of Shigellosis.

Other clinical features including presence of blood in stool, presence of mucus in stool, abdominal pain, flatulence, tenesmus, nausea/ vomiting and fever showed remarkable improvement in test group patients clearly display the result that ShigelDysent is significant in alleviating the clinical features of Shigella infection [Table 3].

\begin{tabular}{|l|l|l|l|l|l|l|}
\hline Consistency of stool & \multicolumn{2}{l}{ Test group frequency } & \multicolumn{2}{l|}{ Control group frequency } \\
\hline & Frequency & Percent & $\begin{array}{l}\text { Cumulative } \\
\text { Percent }\end{array}$ & Frequency & Percent & $\begin{array}{l}\text { Cumulative } \\
\text { Percent }\end{array}$ \\
\hline Bloody mucoid & 16 & 12.8 & 12.8 & 14 & 11.2 & 11.2 \\
\hline Watery & 13 & 10.4 & 23.2 & 15 & 12 & 23.2 \\
\hline Soft & 36 & 28.8 & 52 & 34 & 27.2 & 50.4 \\
\hline Semisoft & 34 & 27.2 & 79.2 & 39 & 31.2 & 81.6 \\
\hline Mucoid & 26 & 20.8 & 100 & 23 & 18.4 & 100 \\
\hline Total & 125 & 100 & & 125 & 100 & \\
\hline Outcome & & & & & & \\
\hline Firm Formed & 87 & 69.6 & 69.6 & 73 & 58.4 & \\
\hline Soft Formed & 29 & 23.2 & 92.8 & 37 & 29.6 & 58.4 \\
\hline Unformed & 8 & 6.4 & 99.2 & 15 & 12 & 88 \\
\hline Semiliquid & 1 & 0.8 & 100 & 0 & & 100 \\
\hline Total & 125 & 100 & & 125 & 100 & \\
\hline
\end{tabular}

Table 1: Consistency of stool in both groups. 
Citation: Qureshi T, Saeed A, Usmanghani K, Asif HM, Zaidi SF (2015) ShigelDysent efficacy in alleviating clinical sign and symptoms prevailing in Shigellosis. Int J Gastroenterol Disord Ther 2: 114. doi: http://dx.doi.org/10.15344/2393-8498/2015/114

Page 3 of 5

\begin{tabular}{|l|l|l|l|l|l|l|}
\hline Presence of blood & \multicolumn{2}{|l|}{ Test group frequency } & \multicolumn{3}{l|}{ Control group frequency } \\
\hline Pre treatment & Frequency & Percent & $\begin{array}{l}\text { Cumulative } \\
\text { Percent }\end{array}$ & Frequency & Percent & $\begin{array}{l}\text { Cumulative } \\
\text { Percent }\end{array}$ \\
\hline Trace & 66 & 52.8 & 52.8 & 78 & 62.4 & 62.4 \\
\hline Fair & 52 & 41.6 & 94.4 & 33 & 26.4 & 88.8 \\
\hline Significant & 7 & 5.6 & 100 & 14 & 11.2 & 100 \\
\hline Total & 125 & 100 & & 125 & 100 & \\
\hline Post treatment & & & & & & \\
\hline Absent & 119 & 95.2 & 95.2 & 82 & 65.6 & \\
\hline Trace & 4 & 3.2 & 98.4 & 100 & 32.8 & \\
\hline Fair & 2 & 1.6 & & 2 & 1.6 & 98.4 \\
\hline Total & 125 & 100 & & 125 & 100 \\
\hline
\end{tabular}

Table 2: Frequency regarding presence of faecal blood.

\begin{tabular}{|c|c|c|c|c|c|}
\hline \multirow{2}{*}{$\begin{array}{l}\text { Sign/Symptoms } \\
\text { Abdominal Cramps }\end{array}$} & \multicolumn{2}{|l|}{ Test } & \multicolumn{2}{|l|}{ Control } & \multirow[t]{2}{*}{$p$ value } \\
\hline & Before treatment & After treatment & Before treatment & After treatment & \\
\hline Absent & $74 \%$ & $91 \%$ & $77 \%$ & $97 \%$ & \\
\hline Mild & $18 \%$ & $9 \%$ & $23 \%$ & $3 \%$ & 0.06 \\
\hline Moderate & $9 \%$ & - & - & - & \\
\hline Abdominal pain & Before treatment & After treatment & Before treatment & After treatment & \\
\hline Absent & $24 \%$ & $97 \%$ & $35 \%$ & $82 \%$ & \\
\hline Mild & $34 \%$ & $3 \%$ & $51 \%$ & $18 \%$ & 0.00 \\
\hline Moderate & $42 \%$ & - & $14 \%$ & - & \\
\hline Flatulence & Before treatment & After treatment & Before treatment & After treatment & \\
\hline Absent & $91 \%$ & $98 \%$ & $69 \%$ & $95 \%$ & \\
\hline Mild & $6 \%$ & $2 \%$ & $25 \%$ & $5 \%$ & 0.31 \\
\hline Moderate & $3 \%$ & - & $6 \%$ & - & \\
\hline Tenesmus & Before treatment & After treatment & Before treatment & After treatment & \\
\hline Absent & $5 \%$ & $98 \%$ & $51 \%$ & $85 \%$ & \\
\hline Mild & $37 \%$ & $2 \%$ & $42 \%$ & $15 \%$ & 0.000 \\
\hline Moderate & $58 \%$ & - & $6 \%$ & - & \\
\hline Nausea & Before treatment & After treatment & Before treatment & After treatment & \\
\hline Absent & $35 \%$ & $92 \%$ & $85 \%$ & $90 \%$ & \\
\hline Mild & $54 \%$ & $8 \%$ & $5 \%$ & $6 \%$ & \\
\hline Moderate & $11 \%$ & - & $6 \%$ & $4 \%$ & 0.46 \\
\hline Severe & - & - & $4 \%$ & - & \\
\hline Anorexia & Before treatment & After treatment & Before treatment & After treatment & \\
\hline Absent & $32 \%$ & $100 \%$ & $70 \%$ & $84 \%$ & \\
\hline Mild & $68 \%$ & - & $12 \%$ & $14 \%$ & \\
\hline Moderate & - & - & $14 \%$ & $2 \%$ & 0.000 \\
\hline Severe & - & - & $4 \%$ & - & \\
\hline
\end{tabular}

Table 3: Clinical signs and symptoms before and after treatment in both groups.

\section{Discussion}

A number of plant extracts and natural products have been shown to work synergistically like that antibiotic activity against resistant strains of Shigella. Shigellosis is one of the most common infections in developed countries where as in the developing countries undergoing industrialization usually exhibit with areas of poor and inadequate sanitation [10]. A three day

Int J Gastroenterol Disord Ther ISSN: 2393-8498 course of ciprofloxacin is the treatment currently recommended by the WHO for shigellosis, including $S$. dysenteriae type 1 infections. The WHO, after considering the risks and the benefits, has recommended ciprofloxacin as the primary treatment of shigellosis in adults and children [11-13]. The current knowledge of the mechanisms and the global distribution of Shigella with reduced susceptibility to ciprofloxacin remain limited. Therefore, due to the 
Citation: Qureshi T, Saeed A, Usmanghani K, Asif HM, Zaidi SF (2015) ShigelDysent efficacy in alleviating clinical sign and symptoms prevailing in Shigellosis. Int J Gastroenterol Disord Ther 2: 114. doi: http://dx.doi.org/10.15344/2393-8498/2015/114

ciprofloxacin resistance, alternative therapies could be the viable option to find out effective treatment of shigellosis. Etiological and clinical features elaborated with shigella infection consist of blood and mucus in stool, cramps, abdominal pain, tenesmus, fever and chills. The ciprofloxacin dose management consists of 500mg ODS for five days is the first line of diarrheal diseases. Moringa stenopetala was one of the said plants assessed for shigella treatment. It was reported that the biological active compounds isolated from both leaves and seeds of the plant by a bioassay guided fractionation exhibited antimicrobial activity against Shigella. In the late 1960s, Shigella suddenly reappeared in epidemic form in Central America and Mexico where it has again become a major public health problem and, more recently, in Asia and Africa including Pakistan the shigella infection is on rise [14]. Inflammatory diarrhea occurs with inflammation of bowel mucosa, which limits its ability to reabsorb fluid and it can occur with Shigella. Until recently, several studies have been conducted on the antimicrobial properties of the leaf and stem extracts of Myrtus communis (myrtle) against pathogenic bacteria and good results have been obtained about its effects on Shigella [15]. The minimum bactericidal concentration (MBC) of Myrtle for most tested microorganisms was similar to the $\mathrm{MBC}$ i.e. $0.5 \mathrm{mg} / \mathrm{ml}$. for Salmonella aureus, $2.5 \mathrm{mg} / \mathrm{ml}$ for Pseudomonas mirabilis and Pseudomonas vulgaris, $15 \mathrm{mg} / \mathrm{ml}$ for Klebssiela spp, Salmonella typhi, and $20 \mathrm{mg} / \mathrm{ml}$ for Pseudomonas aeruginosa. The MBC of Myrtle for the two relatively least sensitive species, Shigella and E. coli was $40 \mathrm{mg} /$ $\mathrm{ml}$ and $45 \mathrm{mg} / \mathrm{ml}$ of media, respectively. The antibacterial activity of Myrtle was markedly increased by 18 times after it has been autoclaved at $1210 \dot{C}$ for 15 minutes [16]. The isolated lectin from the fruit pulp of Aegle marmelos exhibited its effect against Shigella dysenteriae infection. The effect of Aegle marmelos fruit lectin on the adherence of Shigella dysenteriae to human colonic epithelial cells (HT29 cells) was evaluated by Enzyme Linked Immune Sorbent Assay and invasion was analyzed. The protective nature of the Aegle marmelos lectin was assessed by analyzing apoptosis through dual staining method. Aegle marmelos lectin significantly inhibited hemagglutination activity of Shigella and its minimum inhibitory concentration is $0.625 \mu \mathrm{g} /$ well [17]

The anti-diarrheal effect of ethanol extract of the dried fruit pulp of Aegle marmelos was studied on various intestinal pathogens. It showed excellent activity against Shigella boydii, S. sonnei and S. flexneri whereas the activity was found to be moderate against $S$. dysenteriae. The minimum inhibitory concentration against the strains of Shigella was recorded between 250 to $500 \mu \mathrm{g} / \mathrm{ml}$ [18]. Aqueous extract of Aegle marmelos enhanced the susceptibility of beta-lactam resistant Shigella flexneri and Shigella dysenteriae towards beta-lactam antibiotics by altering poring channels. Antimicrobial activity has been shown in the leaves of Aegle marmelos and this study is in consistence with the differential expression of ompC and ompF in multi-drug resistant Shigella dysenteriae and Shigella flexneri [19]. As cited the research work performed in vitro and in vivo anti-diarrheal potential of chloroform extract of the root of Aegle marmelos and was found that the extract was comparable to that of ciprofloxacin and mostly active against the strains of Vibrio cholerae, followed by Escherichia coli (E. coli) and Shigella spp [20]. Phylanthus emblica anti-microbial and cytotoxic activities was reported with the alkaloids fraction showed the strongest inhibitory effect against Bacillus subtilis and moderate inhibitory activity against Salmonella typhi, Bacillus cereus, Pseudomonas aeruginosa, Shigella boydii and Shigella dysenteriae [21]

Int J Gastroenterol Disord Ther

ISSN: 2393-8498
The antimicrobial property of aqueous extracts of Aloe vera, Aloe babrbadensis, Daucus carota, Emblica officinalis, Punica granatum was carried out and to assessed the reason for inhibition of growth of pathogenic organisms by DNA and protein analysis. The effect of four citrus fruit peels extract was compared with antibiotics and the ethanol extract concentrate of Citrus aurantifolia and Citrus limon showed a better inhibitory effect on E. coli and Shigella spp. [22]. As such the coded herbal drug ShigelDysent also contains the above mentioned plants which adequately have been shown to inhibit the Shigella spp. Therefore, herbal formulation based on these medicinal plants upon comparison with ciprofloxacin was found to be effective for the treatment of Shigellosis. However, ShigelDysent showed lesser effects as compared with ciprofloxacin but it does not elaborate side or adverse drug reaction.

\section{Conclusion}

In the present study the predominate species was found to be Shigella sonnei due to contaminated drinking water, then Shigella flexneri and lastly Shigella boydii. ShigelDysent (herbal formulation) is effective in alleviation of clinical features of shigellosis comparable with that of standard drug Ciprofloxacin.

\section{Competing Interests}

The authors have no competing interests with the work presented in this manuscript.

\section{Author Contributions}

All the authors substantially contributed to the study conception and design as well as the acquisition and interpretation of the data and drafting the manuscript.

\section{Acknowledgement}

The authors are thankful to the clinical and non-clinical staff of Shifa-Ul-Mulk Memorial Hospital for Eastern Medicine for the help and support in the collection of data.

\section{References}

1. Tibri ABM (1997) Zaheer (Paichish), Al-Moaljaat Buqratia, Central Council For Research in Unani Medicine 3: 406-407.

2. Azmi WA (1992) Zaheer, Moaljaat-Amraz-e- Hazam wa Toaleed wa Tanasil, Tariqi Urdu Bureau, India, 2: 299-305.

3. AL-Razi MBZ (2000) Amraz-e-Amaa, Kitab-ul-Hawi-Hawi Kabir, Centra Council for Research in Unani Medicine, India 8: 16-21.

4. Batzing BL (2002) Microbial Growth, Microbiology- An Introduction, Wards Worth Group Brooks/cole, p 61, 64,69, 214 269, 270, 363, 476, 477.

5. Tortora JG, Funke BR, Case CL (2001) Shigellosis (Bacillary Dysentery), Microbiology An Introduction, Wesley Longman, 7th Edition, USA, p 691692.

6. Keusch GT, Kopecko DJ (2005) Shigellosis, Harrison's Principles of Interna Medicine-Infectious Diseases, $16^{\text {th }}$ Edition, p 902-906.

7. Kumar P, Clark M (2005) Shigella, Clinical Medicine, Saunders, 6Th Edition, Philadelphia, p 69-72.

8. Sansonetti PJ, Arondel J, Fontaine A, d'Hauteville H, Bernardini ML (1991) OmpB (osmo-regulation) and icsA (cell-to-cell spread) mutants of Shigella flexneri: vaccine candidates and probes to study the pathogenesis of shigellosis. Vaccine 9: 416-422.

9. Qureshi T, Saeed A, Usmanghani K, Asif HM (2014) Clinical Evaluation of Shigeldysent and Ciprofloxacin in the Treatment of Shigellosis. Eur J Biomed Pharma Sci 1: 77-85. 
Citation: Qureshi T, Saeed A, Usmanghani K, Asif HM, Zaidi SF (2015) ShigelDysent efficacy in alleviating clinical sign and symptoms prevailing in Shigellosis. Int J Gastroenterol Disord Ther 2: 114. doi: http://dx.doi.org/10.15344/2393-8498/2015/114

10. Bye WA, Allan $\mathrm{CH}$, Trier JS (1984) Structure, distribution, and origin of $\mathrm{M}$ cells in Peyer's patches of mouse ileum. Gastroenterology 86: 789-801.

11. Neutra MR, Frey A, Kraehenbuhl JP (1996) Epithelial M cells: gateways for mucosal infection and immunization. Cell 86: 345-348.

12. Ram PK, Crump JA, Gupta SK, Miller MA, Mintz ED (2008) Part II. Analysis of data gaps pertaining to Shigella infections in low and medium human development index countries, 1984-2005. Epidemiol Infect 136: 577-603.

13. WHO (2005) Guidelines for the control of shigellosis, including epidemics due to Shigella dysenteriae.

14. Taheri A, Seyfan A, Jalalinezhad S, Naser, Zahedan F (2013) Antibacteria Effect of Myrtus Communis Hydro-Alcoholic Extract on Pathogenic Bacteria. J Res Med Sci 15:19-24.

15. Alem G, Mekonnen $Y$, Tiruneh M, Mulu A (2008) Invitro antibacterial activity of crude preparation of myrtle (Myrtus communis) on common human pathogens. Ethiop Med J 46: 63-69.

16. Raja SB, Murali MR, Kumar NK, Devaraj SN (2011) Isolation and partia characterisation of a novel lectin from Aegle marmelos fruit and its effect on adherence and invasion of Shigellae to HT29 cells. PLoS One 6: e16231.

17. Joshi PV, Patil RH, Maheshwari VL (2009) In vitro antidiarrhoeal activity and toxicity profile of Aegle marmelos Correa ex Roxb. dried fruit pulp. Nat Prod Radi 8: 498-502.

18. Rani P, Vasisht K and Khullar N (2013) Bion-AutoFigurey: An efficient method to check the in vitro antimicrobial activity of Aegle marmelos against enteric pathogens. IIOAB-India IIOABJ 4: 4-9.

19. Rahman S, Parvin R (2014) Therapeutic potential of Aegle marmelos (L.)An overview. Asian Pac J Trop Dis 4: 71-77.

20. Liu P (2012) Composition of Hawthorn (Crataegus spp.), fruits and leaves of Emblica officinalis (Phyllanthus emblica) FRUITS, Department of Biochemistry and Food Chemistry and Functional Foods Forum University of TurkuTurku, Ph.D. thesis.

21. John S, Iyer P (2012) Antimicrobial Activity of Aloe vera barbedensis, Daucus carota, Emblica officinalis, Honey and Punica granatum and Formulation of a Health Drink and Salad Jeswin Philip. Malay J Microbio 8: 141-147.

22. Dhiren N, Leo C, Sarav H, Ganesh M (2013) Efficacy of Citrus Fruit Pee Extracts Against Pathogens Causing Gastrointestinal Disorders. Ind J Novel Drug deli 5:103-107. 